\title{
Generating Anthropomorphic Phantoms Semi- Automatically from Magnetic Resonance Images
}

\author{
Andrey V. Sklyar, Songxiang Gu, Member, IEEE, Michael A. Gennert, Member, IEEE, Michael A. King, Senior \\ Member, IEEE
}

\begin{abstract}
The ultimate motivation of this work is to increase the accuracy of SPECT reconstructions by accounting for the types of patient motions that happen during acquisitions. Assessing the effectiveness of these motion correction algorithms on real patient motions is difficult, since there are currently no numerical anthropomorphic phantoms that accurately simulate nonperiodic deformations such as bending or twisting. We address this problem by providing a way to generate anthropomorphic numerical phantoms of volunteers in different poses, which can be used to simulate imaging procedures in which the patient moves, as well as to assess the accuracy of motion correction algorithms ${ }^{1}$.
\end{abstract}

\section{INTRODUCTION}

In this work, we developed a semi-automated segmentation approach for extracting the heart, lungs, ribs, spine, and the rest of the body (as one structure). In Section II, we describe the motion correction testing approach that motivates this work. Section III goes over some existing phantom generation and motion simulation literature. Section IV briefly describes the engineering hurdles that had to be overcome to make interactive ITK programs. Section V explains the segmentation approach in detail. Section VI describes the segmentation results we were able to achieve with this approach, with a figure of two segmented volumes at the end of the manuscript. Section VII provides our conclusions and discusses the next steps in this work.

\section{Motivation}

Patient motion during SPECT imaging can severely degrade the quality of the reconstruction and the resulting diagnoses [1]. Motion correction algorithms have been and continue to be developed to try to address this issue [2] [3]. As of now, however, there does not exist a direct, quantitative way to assess the accuracy of these reconstructions, since existing

1 This work was supported in part by the National Institute of Biomedical Imaging and Bioengineering Grant No. R01 EB001457. The contents are solely the responsibility of the authors and do not necessarily represent the official views of the National Institutes of Health. numerical phantoms cannot perform the types of deformations a human can when he or she bends or twists, while acquisitions of real moving patients do not provide the ground truth needed for this type of assessment.

We propose to solve this problem by developing anthropomorphic phantoms of the same volunteer in different poses from fast, high resolution MRI scans of the volunteer. By projecting from phantoms corresponding to different poses at different times during the simulated acquisition, we can simulate the patient shifting from one pose to another during the course of the acquisition, as is common in modalities like SPECT [1]. The motion correction algorithm can then be fed the simulated projection data ${ }^{2}$ and the reconstruction can be compared directly to the set of phantoms used to generate it.

\section{BACKGROUND AND RELATED WORK}

A number of anthropomorphic numerical phantoms have been developed from human data in the past. Most of these were made from high resolution MRI or CT scans of a cadaver in one configuration. The organ regions were identified either by manually drawing contours on each slice corresponding to the borders of different organs [4] or semi-automatically using thresholding and morphology [5] [6].

These works resulted in highly detailed and accurate models at the expense of weeks of work [4]. The time required to perform such segmentations makes these approaches prohibitive if multiple volumes need to be segmented.

The NCAT phantom is another realistic phantom generated from the Visible Human Project that simulates breathing and heart beating [7]. Rather than using voxels, organs in this phantom are represented using Non-Uniform Rational BSplines (NURBS). This mathematical representation makes the process of modifying the shape of different structures and simulating motion using spatial transformation straightforward.

In fact, one preliminary way to test the effectiveness of a motion correction algorithm is to generate a set of phantoms by deforming the NCAT phantom and then simulating

\footnotetext{
${ }^{2}$ Motion correction algorithms frequently capture additional data that they can use to estimate the patient's motion. Such data can be acquired while each stationary pose and fed into the reconstruction algorithm at test time.
} 
acquisitions for these different volumes at different times during the acquisition [2] [3]. Unfortunately, these deformations tend to be either very simple, such as rigid body or affine, or specifically tailored to the deformation model used in the motion correction procedure itself. These are good for demonstrating the type of corrections the algorithms should be able to account for. They do not, however, shed much light on how well the algorithms will do with real patient motion and organ deformations.

\section{Interactive Image Processing Programs}

The segmentation approach that we developed uses many different image processing algorithms. We used the Insight into Segmentation and Registration Toolkit for this task, an open source $\mathrm{C}++$ image processing library that provides a vast number of the latest image processing algorithms [8]. We also used the Visualization Toolkit [9], an open source library that implements many visualization techniques, to display the results of the different steps in the segmentation process. Finally, we used wxWidgets, an open source Graphical User Interface library [10], to implement the various types of input widgets used to specify parameter values to the image processing algorithms, with the familiar sliders and textboxes being the most frequently used.

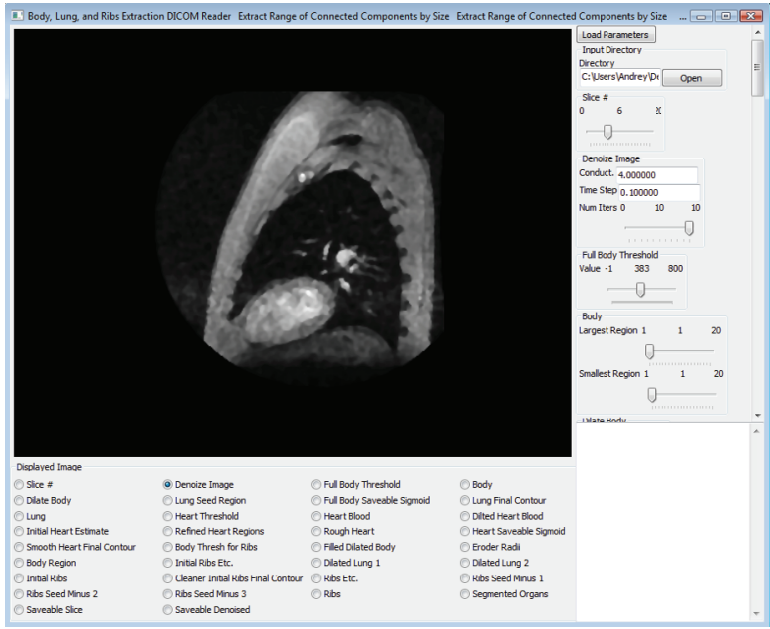

Fig. 1. Interactive segmentation application implemented using ITK, VTK, and wxWidgets.

In writing some preliminary image processing applications using these libraries, we identified a number of issues that were important for writing proper $\mathrm{C}++$ programs, but that were too low level for someone developing image processing application to worry about ${ }^{3}$. As a result, we developed our own high level programming language that succinctly

\footnotetext{
${ }^{3}$ These included the overhead incurred in order to be able to display the output of every ITK filter in VTK, saving and loading parameter values, being able to save the output of each ITK filter, and the specific mechanism by which the values from the sliders or textboxes actually got to the ITK filters.
}

expressed the ideas that are important when developing an image processing application. This language was expressive enough that all the low level details could be generated directly from the high level programs. It allowed us to develop these applications more quickly and reliably than if we continued to program directly in $\mathrm{C}++$.

\section{Segmentation ApPROACH}

We segment the body based on the properties of the organs of interest. In these images, the blood in the heart is bright, the air in the lungs is black, and muscle is gray. The ribs are the dark region contained between the lungs and the soft tissue. This leads to the "threshold and refine" approach for extracting the organs.

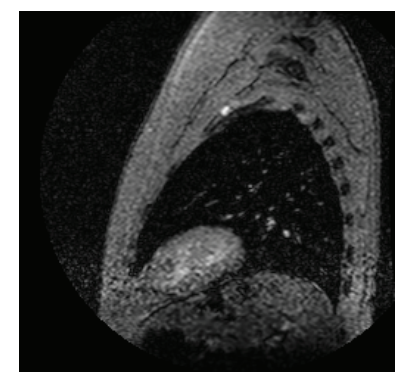

Fig. 2 Input Slice

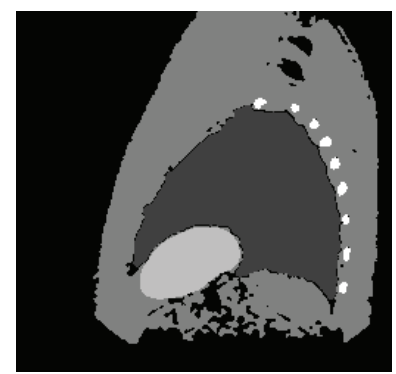

Fig. 3 Segmented Slice
After the image is denoised using anisotropic diffusion [11], sets of connected components are extracted using thresholding. These represent the initial, rough boundaries for the organs of interest. They are subsequently refined using morphology and active contours.

Morphological dilation thickens a boundary while erosion cuts away at it from the borders. Active contours refine a boundary by applying three forces over a specified number of iterations [12]. The balloon force either pushes the boundary outward when it is positive, or shrinks it inward when it is negative. The advection slows the boundary's propagation around image edges, and the curvature force acts to smooth out the boundary.

\section{A. Body}

We extract the body region using thresholding and connected components. The threshold is chosen such that it includes as much as possible of the gray soft tissue in the image, but high enough that the noise in the background does not significantly distort the body contour. The largest connected component is

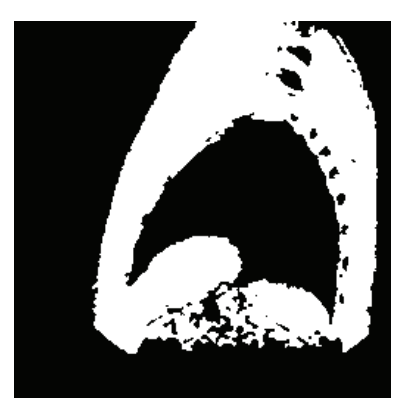

Fig. 4. Body Region extracted and used to represent the body. In later steps, the heart and ribs will be extracted from this region as well. 


\section{B. Lung}

The lung is the black region inside the body. Frequently, this region is connected to the dark region outside of the body due to the dark air in the stomach, the MRI's restricted field of view, and noise present in the image. We close these gaps in the body by dilating it with a structuring element whose radius is selected

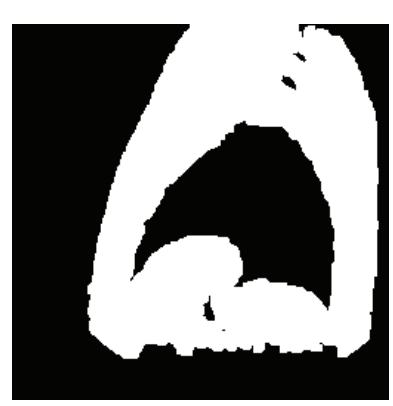

Fig. 6. Dilated Body to be just large enough to close the gaps. This also has the effect of eroding the dark region inside the body

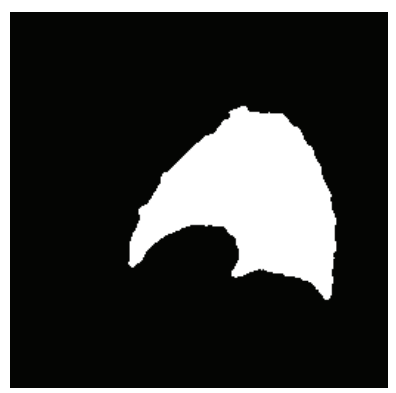

Fig. 7. Lung Region corresponding to the lungs. We blow that region back up using active contours with a positive propagation force. The advection and curvature forces are chosen such that the boundary doesn't leak too much into the ribs, but still gets into the sharp corners above the diaphragm and near the heart.

\section{Ribs and Spine}

The ribs are the dark regions in the body just outside of the lungs. We obtain an initial region for the ribs by extracting the dark region between the lungs and the body within a user specified number of pixels of the lung's border. A

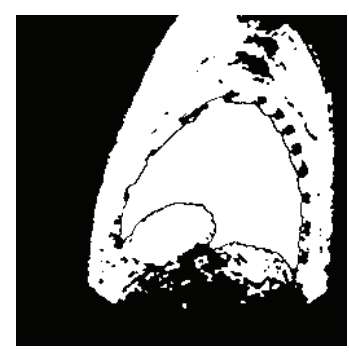

Fig 8. Lung and Body different version of the body is used in this case, thresholded at a higher value in order to expose more of the dark ribs.

This initial region is refined using active contours to smooth out the boundaries. The final ribs are extracted from all the remaining connected components by specifying a smallest and largest size for the ribs and then manually removing spurious features that fall within that range.

Parts of the spine are extracted using the same approach, relying solely on the proximity to the lungs and the darkness of the bones.

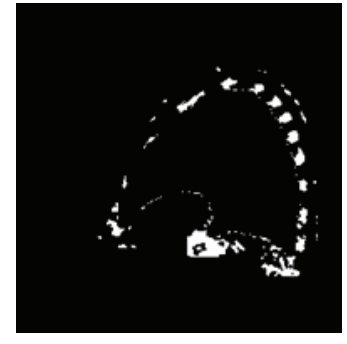

Fig. 9. Initial Ribs

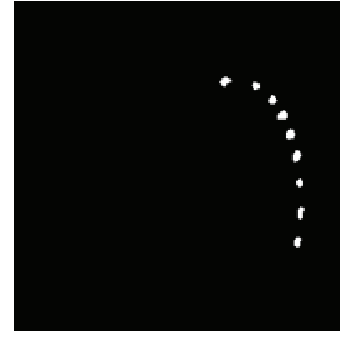

Fig. 10. Final Ribs
D. Heart

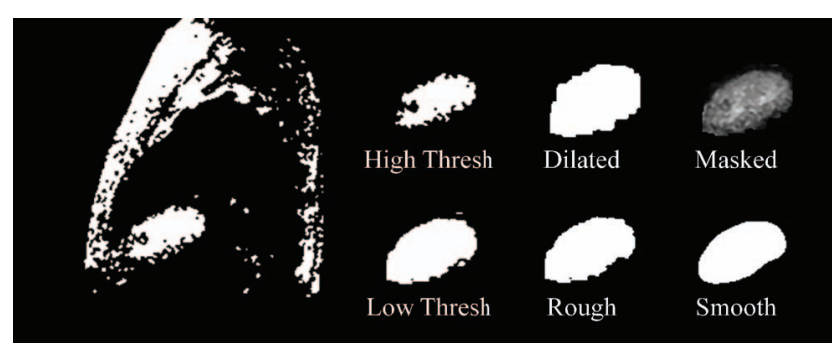

Fig. 11. Heart Segmentation

The blood inside the heart is brighter than the rest of the soft tissue around it. This allows us to use a high threshold value to separate the blood from the rest of the body. We then dilate that region and mask the original input image to extract the tissue around the blood. This allows for a lower threshold to be used, which captures more of the soft tissue of the heart. The largest component is extracted, but the boundary is still rough, so we smooth it out using active contours.

\section{Results}

We tested our segmentation approach on data acquired in an earlier study aimed at assessing the effects of patient motion on the location of the heart [13]. Volunteers held their breath and two cardiac triggered MRI acquisition was performed one after another. This assured that the heart was at the same location in the cardiac cycle in each slice and the lung volume was the same in both poses. The MRI volumes spanned the part of the thorax containing the heart. Each volume was made up of 20 saggital slices, each $4 \mathrm{~mm}$ thick with a $2 \mathrm{~mm}$ gap between slices. Each slices image is $256 \times 256$ pixels, with $1.367 \mathrm{~mm} 2$ pixels.

The segmentation results are provided at the end of this manuscript. Each segmentation took approximately 3 hours.

\section{CONCLUSIONS AND FUTURE WORK}

We have demonstrated the first steps in automating the phantom generation process from MRI volumes of real volunteers. Having a fast and accurate segmentation process is going to be essential for generating the libraries of segmentations that will be necessary for motion simulation and testing.

We can increase the accuracy and reduce the time necessary to segment the volumes by using more sophisticated segmentation and image processing techniques. We can use a database of previously preformed segmentations of the organs and look for them in the most probable places. In the same vein, we can explicitly look for specific structures within the volumes, since, for example, we know there should be twelve ribs in the body that attach to the vertebrae in known locations. This would really help for the thin front ribs, which are missed in most of the demonstrated segmentations. It 
would also complete the spine segmentation we partially accomplish in this work.

We also did not extract other structures present in thorax, such as the scapula, sternum, and collar bone. There also seems to be enough contrast between the blood and the muscles in the heart in these images to extract the heart walls. Some of these structures can be extracted using the threshold and refine approach used in this work, while others will require a more sophisticated approach.

ITK already provides implementations of the algorithms necessary to achieve these improvements. Using the high level programming language developed as part of this work, developing these more sophisticated segmentation applications should be straight forward.

\section{REFERENCES}

[1] Botvinick, Y. Zhu, W. O'Connell, and M. Dae, "A qualitative assessment of patient motion and its effect on myocardial perfusion SPECT images," in The Journal of Nuclear Medicine, vol. 34, February 1993.

[2] B. Feng, H. Gifford, R. Beach, G. Boening, M. Gennert, and M. King, "Use of three-dimensional Gaussian interpolation in the projector/backprojector pair of iterative reconstruction for compensation of known rigid-body motion in SPECT," in IEEE Transactions on Medical Imaging, vol. 25, pp. 838-844, July 2006.

[3] S. Gu, J. E. McNamara, J. Mitra, H. C. Gifford, K. Johnson, M. A. Gennert and M. A. King, "Body Deformation Correction for SPECT Imaging", in Conference Record of Medical Imaging Conference, Nov 2007.

[4] M. Coan, "Voxel-Based Computational Models of Real Human Anatomy: A Review," in Journal of Radiation and Environmental Biophysics, vol. 42, pp 229-235, 2003.

[5] K. Saito, A. Wittmann, S. Koga, Y. Ida, T. Kamei, J. Funabiki, M. Zankl "Construction of a Computed Tomographic Phantom for a Japanese Male Adult and Dose Calculation System," in Radiation and Environmental Biophysics, Mar 2001.

[6] M. Zankl, A. Wittman, "The Adult Male Voxel Model 'Golem' Segmented from Whole-Body CT Patient Data," in Radiation and Environmental Biophysics, June 2001

[7] W. P. Segars, "Development and Application of the New Dynamic NURBS-Based Cardiac-Torso (NCAT) Phantom," Ph.D. dissertation, University of North Carolina, NC, Chapel Hill, 2001.

[8] ITK - Segmentation \& Registration Toolkit, http://www.itk.org/ [Accessed: Oct 28, 2009]

[9] VTK - The Visualization Toolkit, http://www.vtk.org/ [Accessed: Feb 21, 2009]

[10] wxWidgets, http://www.vtk.org/ [Accessed: Jan15, 2009]

[11] R. Whitaker and X. Xue, "Variable-Conductance, Level-Set Curvature for Image Denoising," in International Conference on Image Processing, vol. 3, pp. 142-145, 2001.

[12] V. Caselles, R. Kimmel and G. Sapiro, "Geodesic Active Contours," in International Journal on Computer Vision, vol. 22, pp. 61-97, 1997.

[13] S. Gu, J. E. McNamara, J. Mitra, H. C. Gifford, A. V. Sklyar, K. Johnson, M. A. Gennert, M. A. King, "Pattern Independent Deformation Estimation Illustrated by MRI", in Conference Record of Medical Imaging Conference, Oct 2008.

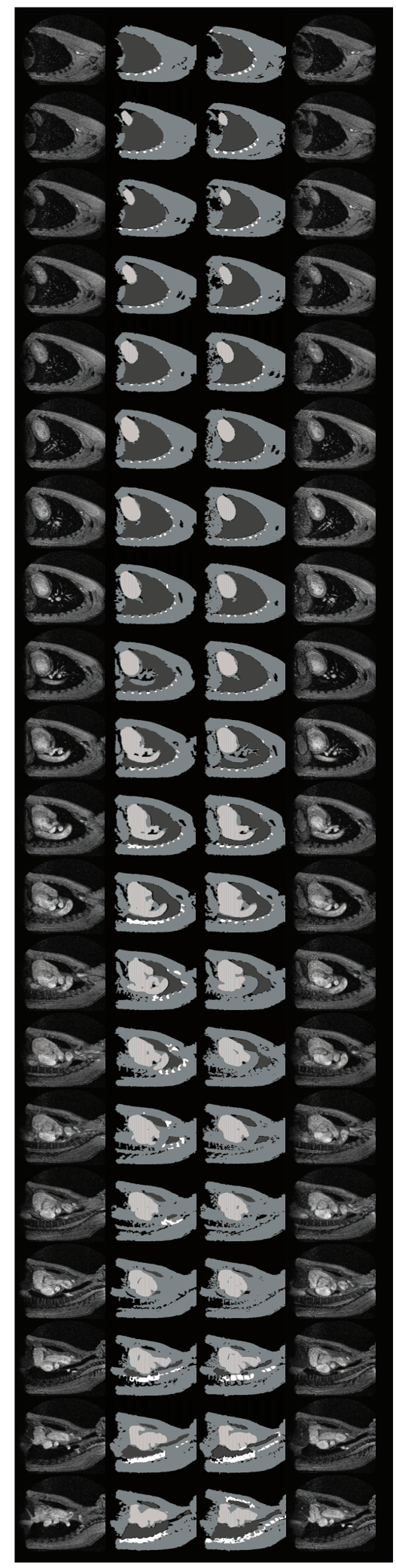

Fig. 12. Segmentation Results. The top (right most) two rows are from the same pose, as are the bottom two. The segmented regions were put close together to allow slice by slice inspection of differences between the two volumes. 